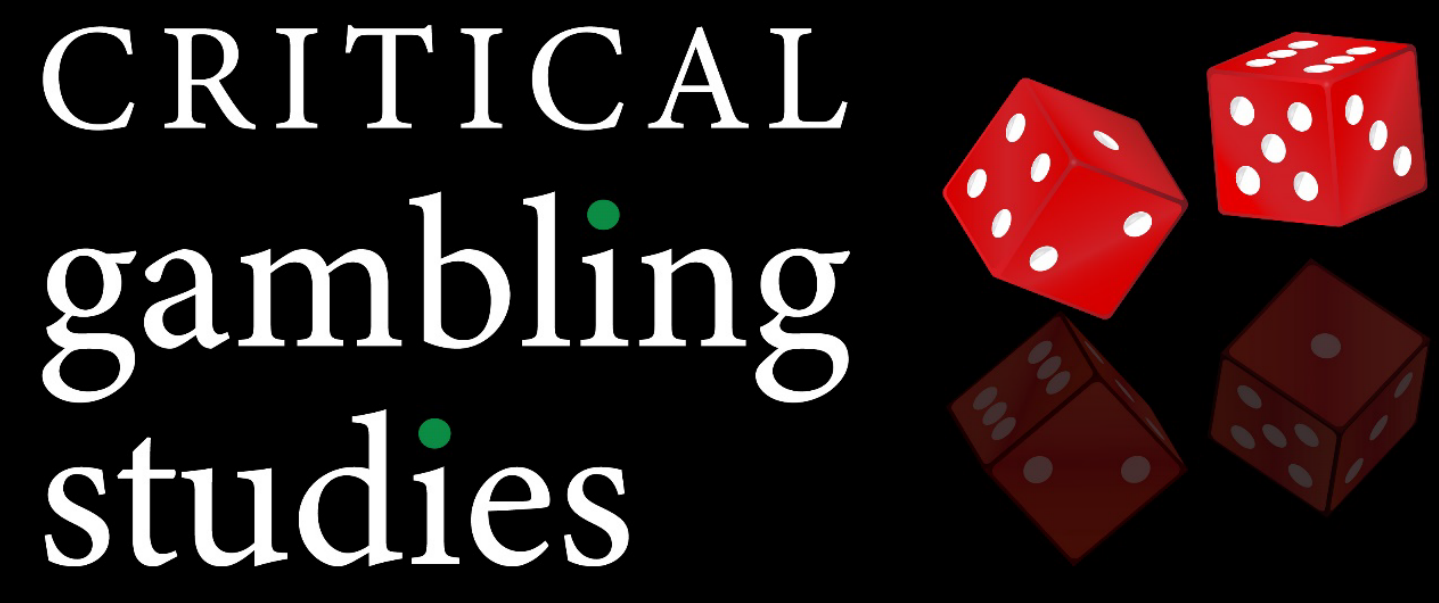

ISSN: 2563-190X. Available Open Access at https://criticalgamblingstudies.com

\title{
The Zone and the Shame: Narratives of Gambling Problems in Japan
}

Eva Samuelsson, Jukka Törrönen, Chiyoung Hwang, Naoko Takiguchi

APA Citation: Samuelsson, E., Törrönen, J., Hwang , C., \& Takiguchi, N. (2022). The Zone and the Shame: Narratives of Gambling Problems in Japan. Critical Gambling Studies, 3(1), 83-95. https://doi.org/10.29173/cgs112

Article History:

Received 2021-06-26

Accepted 2021-12-07

Published 2022-03-07 


\title{
Critical Gambling Studies (2022)
}

Vol. 3, No. 1

What are Critical Gambling Studies?

\section{The Zone and the Shame: Narratives of Gambling Problems in Japan}

\author{
Eva Samuelsson ${ }^{\text {11 }}$, Jukka Törrönen ${ }^{\mathrm{b}}$, Chiyoung Hwang ${ }^{\mathrm{c}}$, Naoko Takiguchi ${ }^{\mathrm{d}}$ \\ ${ }^{\text {a }}$ Department of Social Work, Department of Public Health Sciences/SORAD, Stockholm University \\ ${ }^{b}$ Department of Public Health Sciences/SoRAD, Stockholm University \\ 'Department of Health Promotion and Human Behavior, Graduate School of Medicine, Kyoto University \\ ${ }^{d}$ Department of Sociology, Otani University
}

\begin{abstract}
Japan has one of the highest rates of severe gambling problems in the world. However, the gambling forms that cause the most harm-pachinko and pachislot-are not recognized as gambling in the key legislation. They are understood as entertainment. On the basis of two group interviews with those who have experienced problems with gambling, this study explores how they have dealt with the shame, guilt, and stigma of pachinko-related gambling problems. The narrative analysis shows that the participants carry self-stigma as a result of self-reproach and others' condemnation of their behavior. Feelings of shame, guilt, and fear of being stigmatized have distinctly hindered the process of seeking help. The participants describe how their gambling, which they had attempted to limit, had led to isolation from normal life. The isolation and the failures to control the gambling increased their feelings of shame and destructive behavior. Considering the characteristics of the zone, the loss of self, and the shame, guilt and stigma of failing to control excessive pachinko gambling, it is unreasonable to place the main responsibility on the individual gambler. To reduce gambling harms in Japan and the stigma associated with pachinko and pachislot problems, these gambling forms need to be acknowledged as public health concerns and categorized as gambling in the legislation.
\end{abstract}

Keywords: Japan, pachinko, shame, stigma, help-seeking, narratives

Article History: Received June 26, 2021; Accepted December 7, 2021; Published March 7, 2022

Available Open Access from https://doi.org/10.29173/cgs112

\section{Introduction}

Japan is one of the countries with the highest prevalence of severe gambling problems in the world (Mori \& Goto, 2020) and it has faced vast negative economic, social, and health-related consequences for gamblers and their significant others (Moriyama, 2016). The gambling forms that cause the greatest harms pachinko and pachislot (Toyama et al., 2014; Higuchi \& Matsushita, 2017) - are, however, not regarded as gambling but rather as entertainment. Because the transaction between prizes and money occurs not in the pachinko parlors but in nearby shops, pachinko and pachislot are not treated in the gambling legislation (Penal Code, n.d.; Brooks, Ellis \& Lewis, 2008). Regulation has thereby been lacking, which is one of the contributing factors behind the high prevalence of gambling problems in Japan (Komoto, 2014). The labeling of pachinko and pachislot as "entertainment" is problematic in the sense that it neglects the harms related to such activities, contributes to the division of "capable" gamblers versus "incapable" gamblers, and enhances the stigmatization of those who cannot handle the risks inherent in these activities. Framing gambling as "entertainment" is part of the international gambling industry's ambition to position gambling as harmless fun (Francis \& Livingstone, 2021). Responsible gambling (RG) as a concept was originally developed by the gambling industry as a response to community concerns about the harmful effects of gambling. A tripartite model was proposed where governments, industry, and individual gamblers should share the responsibility to minimize gambling-related harm. But the ultimate "burden of gambling responsibly" is placed on the individual gamblers (Blaszczynski et al., 2011, p. 567). If the problem is perceived to be connected to the product itself, the responsibility would be placed on the producer or provider of the harmful game. If the problem instead is located within the individual consumer as incapable, irresponsible and lacking in selfcontrol, the responsibility and accountability is thereby

\footnotetext{
${ }^{1}$ Corresponding author. Email: eva.samuelsson@socarb.su.se
} 
assigned to the gambler him-/herself. This responsibilization process has the potential consequence that the gambler internalizes the blame (Alexius, 2017), without taking into consideration the gambling industry's role in designing and selling harmful products (i.e., Orford, 2019), the banking sector's inclination to offer fast loans with high interest (i.e., Swanton, Gainsbury, \& Blaszczynski, 2019), and the failure of the government to regulate the gambling market to prevent harm on the population level (i.e., Adams \& Rossen, 2012). Excessive gambling habits and the negative consequences that follow are often associated with feelings of shame and guilt and stigma. Previous studies show that electronic gambling machine (EGM) gamblers are particularly stigmatized (Miller \& Thomas, 2017) and that responsible gambling discourses in fact contribute to stigmatization, by focusing on personal responsibility (Miller \& Thomas, 2018). There is, however, a lack of research illustrating the lived experiences of specifically pachinko and pachislot gamblers, and how they relate to shame, guilt and stigma within a Japanese context. This article aims to fill this knowledge gap.

\section{Pachinko and Pachislot Problems in Japan}

Gambling policy in Japan has been intentionally inconsistent, by banning all gambling apart from lotteries and certain horse, bicycle, boat and motorcycle betting (Penal Code n.d.; Mori \& Goto, 2020) but tolerating an extensive and harmful pachinko industry (Cassidy, 2020). An estimated 3.2 million people (3.6\% of the population) have had severe gambling problems at some point in their lives (Higuchi \& Matsushita, 2017), which is significantly more than in many other countries (Imai, 2018). In the latest national survey, 2.2\% of the population is estimated to have severe gambling problems in the last year (SOGS 5+) (Matsushita, Nitta \& Toyama, 2021). Pachinko and pachislot gambling are the largest leisure activities, with parlors in abundance in Japanese society (Ino, lyama \& Takahashi, 2020). Pachinko is a hybrid of pinball and slot machine where small metal balls are launched into a track possibly releasing more balls. Pachislot is faster, and the gambler can press different buttons to make the wheels stop spinning. The more balls the gambler obtains, the higher the value of the price exchanged. The prizes are commodities (pens, lighters, electronic devices, etc.) that are exchanged for money in adjacent shops (keihin kokan-jo) (Roberts \& Johnson, 2016).

Despite a decline of the pachinko and pachislot industry during recent years, especially during the Covid-19 pandemic when the parlors were required to close, about 7.1 million inhabitants continue to gamble in pachinko parlors, where they generated the equivalent of 132.7 billion USD during 2020 (Japan Productivity Center, 2021). Japan has more gambling machines than any other country in the world (4.2 million, compared to the roughly 900,000 gambling machines of the runner-up, the United States) (Gaming
Technologies Association, 2020), and the sector employs over 220,000 people (Japan Productivity Center, 2020). There are thus strong economic interests to keep pachinko and pachislot gambling legal, despite extensive negative consequences on individual, community, and societal levels.

The most severe consequences from excessive gambling in Japan are social isolation, unemployment, health problems, criminality (Moriyama, 2016), personal bankruptcy, and suicide (Tanabe, 2010). The high incidence of gambling problems among Japanese men could partly be attributed to the stress and alienation experienced as a result of the structural and economic changes in the labor market (Takiguchi \& Rosenthal, 2011). The recent economic recession and the ageing population have created great challenges to Japanese society. The employment conditions and social security institutions have deteriorated since the 1990s (Manzenreiter, 2013). In the absence of an allembracing public social welfare system, poverty and social insecurity have increased (Suzuki et al., 2010). The Japanese welfare state is characterized by a high share of private health care and an expectation that the family and relatives will take care of their members according to a market-oriented family policy model (Lundberg et al., 2008). Government support should be offered only when the family or civil society resources are insufficient (Esping-Andersen, 1997). Seikatsu hogo, the public support by way of economic allowance, housing, or treatment, can be provided only when other alternatives such as savings, property, pensions, financial support from family and other relatives are exhausted (see Ministry of Health, Labour and Welfare, 2019). If an individual needs support, co-habitants, parents, children, and siblings have the obligation to provide for the needs (Saraceno, 2016) and they are asked if they could support the person applying for social welfare. This is one of the main barriers for gamblers to seek help (Takiguchi \& Rosenthal, 2011).

Hospitals and private therapists in Japan offer support and treatment for gamblers and their significant others. Only recently, in April 2020, was cognitive behavioral treatment (CBT) for gambling problems included in the national public health insurance (Kamimura, 2020). The non-governmental 12step-oriented support groups of Gamblers Anonymous (GA) have been active in Japan since 1989 (Takiguchi \& Rosenthal, 2011), and Gam-Anon for concerned significant others since 1991 (Oka, 2013). The twelve steps and traditions have been adapted to suit the Japanese context, making them less confrontational (Takiguchi \& Rosenthal, 2011). Currently, Japan has 204 GA groups (GA Japan Information Center, n.d.) and 188 Gam-Anon groups (Gam-Anon, n.d.). Ads are posted in the pachinko parlors about the national helpline for gamblers to call for information about self-help meetings and treatment centers (Takiguchi \& Rosenthal, 2011). There is nevertheless a lack of available treatment settings in many areas of the 
country, and a great need for staff in health and welfare with competence to offer support and treatment for gambling problems (Moriyama, 2016; Tomida \& Hisanaga, 2020). As a rule, help-seeking rates for gambling problems tend to be low, both in Japan (e.g., Yokomitsu et al., 2020) and internationally, with barriers to help-seeking including shame, problem denial, and lack of treatment availability (e.g., Loy et al., 2019). People with gambling problems commonly report low self-esteem and high levels of stigma. The main strategy to handle the situation is to hide the problems for fear of being exposed, judged, and discriminated against by concerned significant others and care personnel. Affected individuals tend to withdraw and avoid seeking support from family members or the care apparatus (Hing et al., 2016a). The Japanese culture is often described as especially shame-oriented (Scheff, 2013; Tanaka, 2018), with a powerful fear of losing face (Goffman, 1963). In this context, shame and guilt seem to have strong relational characteristics which appear when individuals fail to fulfil their duties and obligations to the family (Song, 2009). According to a recent Japanese national survey, $72.6 \%$ of the respondents felt that in the case of gambling problems, the responsibility rests on the gamblers themselves, whereas the equivalent numbers for alcohol problems $(60.7 \%)$ and depression (8.8\%) were lower (Matsushita, Nitta \& Toyama, 2021). The strong sense of shame, guilt, and stigma connected with gambling and other addiction problems are not exclusive to the Japanese context (see Room, 2005; Yi \& Kanetkar, 2011), but being publicly exposed for committing a crime, being indebted, or borrowing money from the black market to cover one's debts can entail a real risk of social exclusion in Japan, not only for the gamblers but also their family members. Cases of gamblers' arrests have resulted in rejection by the family, and gamblers' children have lost their jobs or been forced to divorce (e.g., Takiguchi \& Rosenthal, 2011). Many Japanese newspapers publish the name, address, and occupation of persons who have been arrested for committing even minor crimes and bringing disgrace to the family. There is a persistent belief that it is impossible to recover from mental health problems, including substance use and gambling problems, and the idea similarly persists that a weak nature lies at the root of the problems (Ando et al., 2013). Concerned significant others express fear of public exposure of their relatives' gambling problems. They worry about losing friends or having to move to another city. The shame and fear of public stigma keep the gambling problems hidden (Takiguchi \& Rosenthal, 2011).

\section{Shame, Guilt, and Stigma of Gambling Problems}

In this study, we approach the gamblers' narratives about pachinko and pachislot gambling in Japan by drawing on symbolic interactionism and by paying special attention to gamblers' experiences of shame, guilt, and stigma. Symbolic interactionism emphasizes that meanings and the self are constructed in interaction with others (Blumer, 1969). The actors are viewed as conscious, purposeful, and self-reflexive, actively shaping their behavior. To understand how gambling, shame, and recovery are meaningful social acts, we need to use methods that enable us to analyze how they emerge in interaction with diverse social and cultural contexts, the specific interview situation, and self-help and treatment settings.

Shame is a complex emotion, its meaning ranging from embarrassment to humiliation. It is often associated with failure and a negative self-image (Scheff, 2003; Thomas et al., 2020). Shame is present in all interactions, serving its purpose by controlling our behaviors and instilling in us an idea of how we should behave (Rose, 1999). Shame is an opposite emotion to pride (Scheff, 1990): while pride indicates secure social relations, the presence of a positive register of feelings where individuals experience themselves as good and important, shame points to threatened social relations, to the existence of negative sentiments in one's relations to others. If pride contributes to the resurrection, maintenance, or thickening of social relations, shame signals troubles in relationships. Pride and shame can be seen as basic emotions in the construction of self, against which the agent mirrors social respect from others (Cooley, 1992)

Moreover, shame can be distinguished from guilt. Shame arises when one breaks cultural and social expectations and norms ("I am a bad person"), whereas guilt arises when one behaves contrary to one's values ("I did something stupid") (Miceli \& Castelfranchi, 2018). In the case of shame, "the others" are viewed as an audience that witnesses our embarrassment, whereas in the case of guilt, "the others" are seen as victims who suffer from our actions (Lebra, 1983). Feelings of shame are generated when our flaws are revealed to others and become the subject of evaluation (irrespective of whether the flaws are real or imagined). Feelings of guilt originate from our own judgment, when, for example, we are not behaving according to our own self-image. However, in everyday conversations we seldom separate shame and guilt: the concepts are often used simultaneously and together (Tangney, 2002).

Experiences and feelings of shame can lead to stigma. Stigma can be defined as a social process occurring when individuals are discredited due to perceived negative attributes, behaviors, or social identities disqualifying them from social acceptance (Goffman, 1963). A distinction is commonly made between public stigma, concerning societal reactions, judgments, and the formation of negative stereotypes toward individuals in the stigmatized condition, and self-stigma, where the individual internalizes the stigmatized attitudes followed by impaired self-esteem (Corrigan, 2004). People with gambling problems often have a negative self-image manifest in low self-esteem and weakened self-efficacy. They tend to have selfstigmatizing attitudes such as disappointment, feelings 
of shame, guilt, humiliation, weakness, failure, selfcontempt, having themselves to blame, and being worse persons than those who can control their gambling (Carroll et al., 2013; Hing et al., 2015). People with substance use and behavioral problems are often subject to stigmatizing processes from concerned significant others, and when in contact with social welfare and health care services (Room, 2005). The public stigma can consist of stereotypes of gamblers as impulsive, irresponsible, irrational, anti-social, unreliable, and unproductive (Hing et al., 2015).

In this study, we explore how recovering gamblers have dealt with the shame, guilt, and stigma related to pachinko and pachislot gambling problems. To produce knowledge on these issues is important, because Japan in general lacks preventive measures against these two gambling forms that cause the most harm. Simultaneously, the welfare system is built upon the expectation that the family has the primary responsibility for protecting and taking care of its members' problems. Excessive gambling also causes substantial stigma for the individual gamblers and their significant others (debt, unemployment, crimes such as embezzlement, theft, fraud, etc.). Despite a number of studies with epidemiological or psychiatric perspectives on gambling (e.g., Akiyama et al., 2019; Horiuchi et al., 2018; Yokomitsu et al. 2019), there is a lack of research from a social (Takiguchi \& Rosenthal, 2011) and qualitative point of view giving Japanese gamblers a voice.

\section{Materials and Methods}

The material for this article was collected during October and November of 2019. Two group interviews were held with eight gamblers, seven men and one woman (aged between 30 to 60 years), in gamblingspecific self-help and treatment settings in urban areas of Japan. Moreover, participant observation was conducted in three different sessions with twelve-step, CBT, and self-help orientations to get insights into the formation of gambling treatment in Japan. The interview guide contained questions about the nature, development, and consequences of the participants' gambling habits, help-seeking experiences, and feelings of shame and guilt. All procedures in the study were performed in accordance with the ethical standards of the 1964 Helsinki Declaration and its later amendments. The participants were given written and oral information in Japanese about the aims, methods, and funding of the study, the voluntariness of participation, and their right not to answer specific questions, and withdraw their consent at any time without reprisal. They were also informed about confidentiality, anonymity, and where to obtain the results of the study. The third and fourth authors aided in access to the interview settings, translation of the information, interpretation, and interviewing.

The group interviews lasted for about 1.5 hours each and were audio recorded. The recordings were transcribed and translated, where the translator somewhat adjusted the language to facilitate the flow in the accounts. The interview material was then processed in an empirically close narrative analysis, with a focus on the content of what was said, rather than how it was said (Kohler Riessman, 2006).

In our narrative analysis, we particularly consider what kinds of feelings of shame, guilt, and stigma contribute to the development of gambling problems, how these feelings become prominent as the gambling problems escalate, and how they act as barriers in the recovery process.

To begin with, we analyze how the gambling started and then proceed to examine how gambling, as it escalated, made the participants isolate or turn away from their primary relations and activities, implying negative feelings of shame, guilt, and stigma. This is followed by an analysis of how gambling, which had led to a crisis and had been revealed, became explicitly connected to the feelings of shame, guilt, and stigma. Finally, we analyze how these feelings prevented the participants' efforts to seek help and begin recovery. In order to give the reader a more concrete picture of the participants, while ensuring their anonymity, the participants' names have been changed and their age is given as an approximate figure.

\section{Results}

\section{How it Started: Getting into the Zone}

The pachinko parlors offer a bright, loud, smokefilled environment with long arrays of electronic gambling machines surrounded by chairs for gamblers (Ito \& Crutcher, 2014). The machines can be described as monstrous hybrids combining electro-mechanical pinball machines with digital slot machines (Rockwell \& Amano, 2019) where the cacophony of the machine and parlor, the interaction with the silver balls and the characters and music from Japanese popular culture, create a space of solitude, with an absorbing form of play (Brooks et al., 2008; Ito \& Crutcher, 2014). As products the electronic gambling machines are designed to disconnect the gamblers, to make them lose track of time and money, thereby transforming entertainment into entrapment (Schüll, 2012). One motive for pachinko players is to unwind from stressful jobs, spending several hours in the parlors to relax after work (Cassidy, 2020). For the participants of this study, gambling initially meant something fun and relaxing. It provided a sense of freedom and recreation but soon transformed to signify an escape from troubles.

I went to the pachinko parlor to celebrate after the graduation ceremony. From that moment I felt that I could do as I wanted without anyone getting angry with me. I gambled, at the most, every day. (...) In the beginning it was really fun and relaxing. I felt better from gambling. (...) When I gambled pachinko for the first time, I knew at once that it suited me and my personality well, and that it could 
be my lifetime hobby. (...) At the pachinko parlor time would fly. Therefore, it was a perfect way to spend spare time. (...) If something bad happened I wanted to gamble. When I filled my head with pachinko data, I forgot the problems. When some time had passed, I felt somewhat relieved. Pachinko was fun and let me forget my problems. I felt there was nothing better than pachinko. (Daiki, 30-40year-old man)

Daiki felt that gambling in the pachinko parlor "was a perfect way to spend spare time" and that it made time fly. Gambling sucked him into its world, made him forget all his problems and he felt living to the fullest in that moment. This is what Natasha Dow Schüll calls "getting into the zone" - an experience also described as "being in the eye of a storm" (Schüll, 2012:2). Yuki's narrative clarifies this experience further:

In the pachinko parlor, there's a lot of noise and the machines twinkle and sparkle everywhere. When I gambled I was very concentrated and could be alone. I could forget bad things that had happened during the day and could be alone. Before I started gambling, I decided how much money to spend. But I gambled more often and for a longer time, resulting in spending more money. (Yuki, 40-50year-old man)

As this passage from Yuki's narrative shows, when a gambler gets into the zone, the outside world disappears. The noise, the constantly flashing lights, and the design of the gambling machines facilitate the loss of time and space and the exclusive focus on gambling. The zone offers a harmonious state where everything else, including the risks of gambling, fades. The gambler no longer gambles to win or to compete with other gamblers. The gambler continues to gamble to stay in the zone as long as possible, which is encouraged by the constant feedback from the machine (Schüll, 2012). In this way, getting into the zone paradoxically offers the gambler a sense of control and peace against the disorder of everyday life that appears out of control and depressing. The passages from Aiko's and Riku's narratives exemplify the process:

I gambled to ease the daily stress. I had constant arguments with my children and went to the pachinko parlor to get some peace and quiet. I was enchanted by the screen of the machine and it was exciting. When the game started, I was free from troubles and relaxed. (Aiko, 50-60-year-old woman)

It was the excitement that I liked, and the performance when I won. It was a dark period of my life and it's difficult to remember. (...) When I was little I had a bad home environment and I thought it was my parents' fault. I wasn't happy with my life, my home, myself, or the lifestyle. Then I learned to play pachinko. When I won, I got a lot of money and was satisfied. I thought that if I continued to win money, my life could change and all the things in my life that I was not pleased with would change. (Riku, 40-50-year-old man)

These quotations demonstrate how getting into the zone freed the participants momentarily from everyday life problems and made them feel restful, alive, excited, and hopeful. In the zone the constraints of space and time disappeared and the participants were relieved of the intersubjective expectations of others (Schüll, 2012). The enchantment of the zone as opposed to the disorder outside it escalated the participants' gambling problems.

\section{Turning away from Primary Social Relations and Activities: Loss of Control and Self}

In the following quotation, Daiki describes the consequences of his escalating gambling problems, which made him turn away from friends, family, and social events and led him to disregard such basic needs as food and sleep.

Over time, the gambling hours and stakes increased extremely. (...) The more I tried to decrease the gambling the more I longed for it. I got used to running away from my problems. It was a huge problem. (...) I didn't know any other way to solve my problems. (...) I prioritized gambling above all else, so I didn't have time to eat or sleep. The most valuable thing in my life by then was gambling. (...) I didn't want to meet anyone. (...) My values became abnormal. I neither went to weddings nor funerals. (...). I completely lost it and that was the worst effect of the gambling. (Daiki, 30-40-year-old man)

We can interpret that Daiki's isolation refers to the increasing presence of shame and guilt in his life. By prioritizing gambling and by dodging encounters that could stigmatize him, he cut himself off from normal human interaction and needs. The next passage from Aiko's narrative illustrates this experience further:

I didn't spend money on the most important things in life, such as food, rent, or clothes, but prioritized gambling. I spent the whole paycheck on gambling and wondered how I would survive to the next salary. I'm a woman and as long as I have wheat flour I can manage somehow. I bought such things and managed if I had water. That's how I lived. (Aiko, 50-60-year-old woman)

If Daiki and Aiko do not explicitly acknowledge that their isolation process with escalating gambling was accompanied by feelings of shame and guilt, Riku expressly recognizes that when he lost control over his 
gambling, he also lost his pride, sense of responsibility, and normal self. As a result, he self-stigmatized himself outside the human world, beyond shame.

When I was stuck in the gambling, I didn't care about other things, for example taking responsibility or doing other regular things. I lied about so many things and nothing made any sense. I stopped caring about my hygiene and more and more other things. Before I started to gamble, I cared about my appearance and how others perceived me. But that feeling was gone. When the gambling influenced my everyday life, I became desperate to fix it. But I thought that if I could win in pachinko and get more money, it would all be OK again. It became a vicious circle. I kept less money in my wallet, found other hobbies, and asked my family to handle my money, but I didn't succeed. (Riku, 50-60-year-old man)

Similarly, Kaito describes himself as "a bad person" and "immature," that he felt it would not matter if he died. Koki felt "worthless" over having failed his family. The participants cite numerous attempts to decrease gambling on their own, by limiting the time, leaving the credit card at home, just bringing a smaller amount of money to the parlor, or keeping themselves constantly occupied with other interests. When these attempts failed, the gambling escalated and led to desperate measures to solve the situation in a vicious circle of debts, lies, shame, and indifference with severe negative consequences (relationship problems, loneliness, unemployment, homelessness, and suicide attempts). In Kaito's case it led to the loss of home and work.

The gambling affected my life in the sense that I didn't want to spend money on something that I didn't really need. I became cheaper and cheaper and in the end I lived in my car. Because of my situation I couldn't rent an apartment, so I had to stay with my family. But if I went home there was a bad atmosphere. Therefore I chose to live in the car, to avoid going home. (...) Eventually I stopped going to work. I only thought of how to get money and felt like I didn't have a life. When I couldn't borrow money from the bank anymore, I didn't hesitate to borrow from the black market. I lost a normal way of thinking (Kaito, 30-40-year-old man)

The examples of this section show that when gambling escalated and became the primary mission in life, the gamblers lost their sense of self, turned away from normal interaction, and started to act against the norms and values of their society. Despite numerous attempts to limit their gambling, the interviewees describe falling deeper into a vicious circle of desperation and lies.

\section{Recognizing Feelings of Shame, Guilt, and Stigma}

After their gambling problems had been discovered or the gamblers had developed a distance from them, the participants were able to explicitly relate their gambling problems to feelings of shame, guilt, and stigma. For example, Yuki says that after realizing what he had done, he felt ashamed of lying and having placed himself and his family in a difficult economic situation.

I was so ashamed of having borrowed several million yen and becoming destitute. It was so embarrassing to talk about but despite that I continued to gamble pachinko. (...) This behavior affected me and I didn't dare to talk about completely ordinary things that I wasn't ashamed of. (Yuki, 40-50-year-old man)

The feelings of shame were often complicated by the fact that when their significant others got to know about the gambling problem, they condemned, disgraced, and stigmatized the gamblers as bad persons-as in the following passage from Riku's narrative:

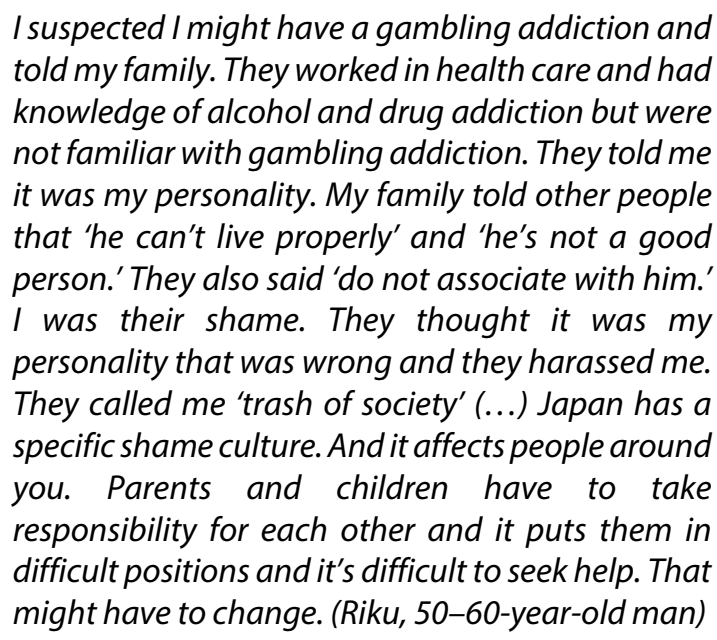

When reasoning about the mechanisms behind the stigma, Riku describes the Japanese culture as built upon mutual obligations toward one another and as especially marked by shame which envelops the whole family system, rather than just the individual. However, not all participants acknowledge feeling ashamed of their gambling, which the passage from Daiki's narrative below exemplifies. Instead of feeling ashamed, Daiki felt guilty:

I never felt shame. I didn't care about other people that much, and therefore I stopped having contact with my friends. Still, I don't feel shame for my gambling. I think it was my family who felt ashamed of me. If your son has a gambling addiction and large debts due to the gambling, you feel ashamed. I understand it and it makes me feel extremely guilty but not ashamed. I chose to 
gamble, which is why I don't have to feel guilty over it, but I feel guilt toward my family who needed to pay my debts or because they felt ashamed of me. There I feel guilty. (Daiki, 30-40-year-old man)

By denying ever feeling ashamed and by emphasizing that he chose to gamble, Daiki may aim to present himself in the interview situation as a responsible person, taking accountability for his actions. However, his description of how "he stopped having contact" with his friends implies that on some level his action was influenced by shame: In order not to make himself vulnerable to social stigmatization and to the feelings of shame, he walked away from his friendship and family ties.

Aiko, again, openly acknowledges feeling ashamed of her gambling. She feared that if her neighbors and friends got to know what she was doing, they would consider her a bad parent. She also felt guilty about lying to her children. The discrepancy between her own actions and what she wanted to teach her children increased her feelings of shame and guilt and made her blame herself as being a bad mother.

I wanted to be a good mother but it was a shame that I gambled pachinko. I didn't want others to think 'she gambles pachinko although she is a single parent' or 'she lies and tells her children that she's working but she's gambling.' I lied too much and it made the children sad. (...)I told the kids that they shouldn't lie (...) but I did the opposite and lied and made them sad. The children's father gambled too and we divorced due to his debts and infidelity. The children witnessed it and thought it was a bad situation. But I did exactly the same thing and felt guilty. (Aiko, 50-60-year-old woman)

When reasoning about the link between gambling problems and shame, the participants distinguish between how different forms of addiction problems are perceived by the general public. While mental health problems are regarded as afflicting individuals undeservedly, without being attributed to a fault of their own, and alcohol problems are attributed to the substance as such, excessive gambling is framed as something in which people rationally and irresponsibly choose to indulge. It is therefore judged as more shameful.

You're affected by mental illness because of different preconditions. Alcohol problems are the same. But gambling you have to take initiative to begin with. You do it by yourselffrom the beginning to all the debts, and it causes feelings of shame and guilt. (Ryusei, 30-40-year-old man)

Mental health problems are somehow accepted, and it is possible to discuss them with your employer. Based on Koki's reasoning below, gambling problems are difficult to disclose out of fear of the humiliation and discrimination that such an exposure would bring.

If I had a mental illness and told about it to the company where I work, it would be regarded as a disability. Therefore it's not shameful. But there's no company in the world that would hire someone who is open about their gambling addiction. In that sense, society reacts differently toward gambling addiction, I believe. (Koki, 30-40-yearold man)

\section{Shame, Guilt, and Stigma in the Process of Help- Seeking and Recovery}

The entanglement of gambling with feelings of shame, guilt, and stigma hindered or complicated the process of help-seeking and recovery for the participants as in the case of Kaito, whose feelings of shame and guilt over his gambling prevented him from seeking help.

I was the biggest obstacle myself. Shame or guilt stopped me from seeking help. If I'd been able to ask for help, I would've felt better. I felt awful but might have been able to talk about it sooner. But instead, I tried to solve the situation in the wrong way, which led to increased shame and guilt. (Kaito, 3040-year-old man).

Similarly, the shame and lack of knowledge of what kind of treatment was available acted as obstacles to Aiko's help-seeking.

I couldn't ask for help because I was so ashamed due to my age. I should be mature. And I didn't know where to get help. I was physically healthy and had no contact with health care. I didn't know how to seek information. (Aiko, 50-60-year-old woman)

Riku blamed himself for the problems. He considered himself weak and cowardly. He also lacked knowledge of gambling problems and did not know where to turn for support. These were the primary obstacles for his help-seeking. Eventually the situation became unsustainable. He did find information about a treatment center, but was reluctant to go there, his family was against it, and he relapsed. In retrospect he wishes that the care personnel had had more knowledge about gambling problems, in order to refer him at an earlier stage.

The reason I didn't seek help sooner was that I had no knowledge about gambling addiction and I didn't think it was a disease. I thought the reason was my bad personality and that there was nothing that could cure it. I didn't think there was any support. (...) But when I couldn't live like normal people, I searched the internet and found this place. 
I wanted to come but my family was against it. It took time to come here. I canceled the first interview and went to the pachinko parlor instead. (...) The biggest obstacle for seeking help was myself. My weakness. I wasn't brave. (...) If the doctor in the health care services had known about gambling addiction, he could've sent me to therapy, but he just kept on asking the same questions over again. (Riku, 50-60-year-old man)

In hindsight Daiki also acknowledges that the primary barrier to help-seeking was his own and the general public's lack of knowledge of gambling problems. This lack of knowledge causes self-blame in those in need and in others it feeds a judgmental attitude. He resists the view that gambling problems have a moral nature, where the root is considered to be the individual's weak character.

The main barrier for seeking help was ignorance. Both my own and among the general public. (...) People believe that the personality is the problem. They believe that if you'd make a real effort, you could solve it. That's why you can't ask for help. If you tell your family about your gambling addiction to get help from health care services, they get really angry with you. You don't know where to turn to or if it actually is a real problem. Such ignorance is the most difficult obstacle to overcome. If l'd known it was a disease I would've dealt with it much sooner. (Daiki, 30-40-year-old man)

Daiki's account illustrates how gambling problems are not taken seriously, in part because they are not seen as legitimate health issues that require support and treatment. The medical model of gambling problems as a disease helps Daiki understand the ambivalence he has experienced. The participants argue for the need of increased knowledge of gambling problems, and the connection with substance use problems and mental health issues, not only in health and welfare services, but also in society at large. Koki also argues for the importance of changing the gambling policy in Japan.

In Japan it's very easy for gamblers to gamble. Even if the government imposed strict age limits and limited the gambling possibilities, the most important thing is to restrict loan possibilities. (Koki, 30-40-year-old man)

Our participants' narratives also testify that sometimes families and significant others were not paralyzed by shame and stigma but were able to help the gambler to treatment. For example, Yuki found his way to treatment thanks to his wife. The following passage from Ryusei's narrative demonstrates how his parents made sure that he went to treatment. By attributing to him a stigma of not being trustworthy, they monitored that he actually participated in treatment and did not go to the pachinko parlor:

My parents accompanied me in the first month. Eventually just my mother. But even if I just had 100 yen, I bet that money. They couldn't leave me alone. They followed me for several months. There was no other way. (Ryusei, 30-40-year-old man)

For the interviewees in this study, the process of help-seeking has been thorny and has involved several stages. When taking the step to seek help in GA meetings or in gambling-specific treatment centers, the participants value being met in a warm atmosphere of acceptance and openness. In the initial phase, they find it crucial to be offered concrete help with handling the economy to facilitate abstention from gambling and unburden the relatives. As shown by Yuki's account below, the participants appreciate a non-judgmental approach among the staff and the other clients that was free of shaming and was able to address the stigma in a liberating way. Repeatedly telling his story helped Yuki reflect on his life and had a healing influence.

I talked to the therapist who said 'come here tomorrow' and then I came. But I was still worried at first. Here no one accuses me when I talk about my problems, my gambling, or my childhood. Even though I reveal bad or awful things that I've done, nobody accuses me. Therefore I could talk and when I talk more I can look back objectively. (Yuki, 40-50-year-old man)

\section{Discussion}

This study has explored how recovering gamblers have dealt with shame, guilt, and stigma related to pachinko and pachislot gambling problems. The gambling has had vast negative consequences for the gamblers themselves and their concerned significant others-economically, emotionally, and socially. Our analysis suggests that excessive pachinko gambling that leads to isolation and destructive behavior constitutes a major social stigma for the gamblers and their families. Despite this, pachinko gambling is still considered entertainment in Japanese legislation. The findings from this study show that the pachinko parlor has symbolized a zone or a sense of freedom for the participants, offering a sanctuary from everyday hardships. In accordance with the ideal of the late modern society, our consumption of goods and services is expected to occur in a rational and self-restrained way (cf. Reith, 2007). Gambling as an activity inevitably involves a risk as an integral part of the appeal. The gamblers thus balance on the fine line between risktaking and self-discipline. As the pachinko parlor offers a zone where it is rational to continue gambling (see Schüll, 2012) to offset the stress and pressure for success we feel in our everyday lives, getting into the zone acts as a platform that facilitates the escalation of gambling 
and the emergence of addiction (Törrönen, Samuelsson \& Gunnarsson, 2020).

Our participants' narratives reveal not only the loss of the self in the gambling moment, but also in relation to priorities in everyday lives. The gamblers isolated themselves from normal life and described experiences of having run up excessive debts, stealing from their concerned significant others or the company where they worked, and in different ways acting contrary to their values and losing their "normality", or their selves. When they failed to control their gambling, and through their actions violated their own and others' values, the result was shame, guilt, self-stigma, and condemnation. The shame is related to gambling as such, as well as being related to the indebtedness, the lies, or being considered a bad person and a disgrace to the family. The experience of shame and guilt is inevitably interconnected, because our self and our behaviors are balanced between what we want to display in front of others (omote $=$ front, the public self) and what is hidden from others (ura = back, the private self) (Doi, 1986). Some of the participants are prone to expressing feelings of guilt over their actions, while others rather express intense feelings of shame over who they have become, internalizing a self-stigma of themselves as "immature," "irresponsible," and "worthless." The self-stigma is created in interaction with the public stigma of gambling problems, in relation to significant others and societal reactions. For example, when gambling disorder was recently included in the national health insurance in Japan (Kamimura, 2020), it was met by public criticism and the argument that people with gambling problems have themselves to blame for their predicament (Tomida \& Hisanaga, 2020).

The participants of our study perceive gambling problems as especially shameful, as they have affected the family economy and are attributed to the individual's weak character and inability to make rational choices. This is in line with previous research: Australian gamblers perceive gambling problems as more stigmatized than alcohol problems, obesity, schizophrenia, depression, and cancer, but less stigmatized than narcotic problems (Hing et al., 2015). Notions of mental health and addiction problems vary through time and space with different levels of moralization (Raylu \& Oei, 2004; Room, 2005). The moral perspective on the individual gambler as a person with a weak character, which prevailed in the early 20th century has broadly been replaced by a medical perspective. But moral views of gambling problems still persist. They appear in the participants' narratives of self-stigma and condemnation by their significant others, as well as in the Japanese public debate. In light of the actual risk of stigmatization of people with gambling problems in Japan, it could be regarded as rational for gamblers to despair and hide their problems.
Gambling problems are to a high degree shaped by social, relational, and cultural circumstances. Decisive life events such as separations, unemployment, or illness in the family can create a situation where the gambling takes precedence in a person's life (Samuelsson, Sundqvist \& Binde, 2018). Despite this, inherent moral (individual deficits) or medical (psychiatric disorders) characteristics of the individual are often emphasized in research and public opinion as explaining gambling problems. In the interviews, the participants described the social circumstances of their gambling story, mainly making use of the disease perspective in their understanding of the problems in relation to family and treatment settings. The gamblers' self-perceptions are inexorably linked to their perceptions of what others believe about them, contributing to their self-stigma. To be able to recover from gambling problems, the gamblers' view of the self needs to be restored, and the disease perspective of addiction offers a credible model. To perceive gambling problems as a compulsive disorder has the potential to decrease the stigma of excessive gambling behavior, for a sick person is not regarded as responsible. But due to the prevailing expectations of rational consumers in the late modern society, choosing to spend time and money on something as unproductive as pachinko gambling is frowned upon. Failure to handle money in a socially acceptable way entails a decisive violation of social and cultural norms. It runs contrary to the ideals of responsible, self-restraining, controlled, rational consumers of late modern society (Rose, 1999) and therefore causes strong feelings of shame and guilt. In Japanese society, where the welfare system is built upon the family as the primary unit of caretaking, the verdict against those who fail to live up to the expectations and responsibilities of the family can be notably harsh.

The participants describe numerous attempts to limit their gambling, through restrictions of time or money spent on gambling or trying to turn to other activities. Their failures have led to increased feelings of shame and destructive violations to try to solve the situation. It is unreasonable to place the main responsibility on the individual gamblers and their significant others to reduce gambling harms, considering the characteristics of the zone, the loss of self, and the shame, guilt, and self-stigma of failing to control excessive gambling behaviors. Based on her extensive fieldwork on gambling in different parts of the world, Cassidy (2020) contends that the idea of responsible gambling fits well with Japanese legislators' ambition to ensure that casinos can bring profits into their regions. By ensuring that gambling is safe for everyone except for the addicted gamblers who have a brain disorder, the responsible gambling discourse continues to place the responsibility for the extensive harms from gambling on the individual consumer (Cassidy, 2020). This responsibilization of the individual gambler (Alexius, 2017) further enhances the stigma of 
those who fail to control their excessive gambling habits (Miller \& Thomas, 2018).

\section{Implications for Support and Treatment}

Our analysis shows how excessive gambling is entangled with the feelings of shame, guilt, and stigma that feed the escalation of problems and push the gamblers toward isolating themselves from normal relations and activities because of the need to hide the problems. Shame, guilt, and stigma constitute tangible obstacles to seeking help from significant others, selfhelp groups, or the care apparatus (see also Suurvali et al., 2009; Yi \& Kanetkar, 2011). To remove obstacles of help-seeking and to limit gambling-related harms such as substance use problems, homelessness, suicide, and other mental health problems (Tanabe, 2010; Yakovenko \& Hodgins, 2020), it is crucial to decrease the associated stigma. Support and treatment should be more readily available through anonymous and digital options, and the public and professional understanding of the nature of such problems needs to be increased (Hing et al., 2016b).

Non-judgmental approaches toward gambling problems, among counselors, are crucial to help gamblers overcome their stigma and facilitate their recovery. There is a need to develop treatment methods that specifically address shame and stigma, help the gamblers re-establish their sense of self-worth and support their need to rebuild their identity, to reconstitute their primary relationships, and to learn functioning coping and problem-solving strategies (Komoto, 2015). As the problems affect the whole family, the strategies need to be designed to also meet the needs of significant others. Employers have a responsibility to detect mental health problems among their employees. This responsibility should be expanded to cover addiction problems in general and gambling problems in particular.

\section{Policy Implications}

To meet the concerns of increased gambling problems in the wake of the opening of international casinos in Japan (Tomida \& Hisanaga, 2020), the government launched a three-year-plan (2019-2021) to implement responsible gambling measures (Government of Japan, 2019; So et al., 2019). The plan includes measures such as removing automated teller machines from pachinko parlors, replacing gambling machines with less stimulating machines, education of parlor staff, and distribution of leaflets and slogans on "secure pachinko/pachislot gaming" (Government of Japan, 2020). The strategies are, however, to a large extent based on voluntary measures of the pachinko industry or place the main responsibility of, for example, voluntary self-exclusion, on the individual gambler (Higuchi, 2020). By directing attention away from structural aspects and policy (e.g., reducing availability of gambling products) towards individual measures (e.g., introducing high-risk limits) (Akiyama et al., 2019), the social problems caused by excessive gambling are reframed as individual pathological deficits (Campbell \& Smith, 2003). Placing the main responsibility on the individual gambler legitimizes the limited state regulation of the gambling market (Reith, 2007) and enhances the stigma associated with "irresponsible" gambling (Miller \& Thomas, 2018).

The fact that pachinko parlors are omnipresent in Japan, commonly in close vicinity to commuting stations and entertainment districts in big cities as well as in suburbs and rural areas with generous everyday opening hours (Manzenreiter, 2013), is evidently problematic from a public health perspective. Substantial harm is imposed on vulnerable populations, i.e., shown by studies indicating that accessibility to pachinko parlors is positively associated with gambling problems in low-income areas (Kato \& Gato, 2018). In Japan, the government encourages the industry to give money directly to private treatment facilities with little awareness of a conflict of interest, thereby legitimizing inadequate and insufficient measures from the gambling industry.

During recent years, there have been signs of decreasing prevalence of gambling participation (Japan Productivity Center, 2020), possibly related to youth preferences for online gaming, less stimulation in electronic gambling machines, and the introduction of upper limits and self-exclusion of consumption loans (Government of Japan, 2020). But rather than directing responsible gambling measures toward individual gamblers, there is still a clear need for increased population-level prevention of gambling problems in Japan. Such universal measures could include limiting access to gambling opportunities and restricting marketing, credits, and loans (see Swanton et al., 2019), or imposing demands of identification (see, e.g., Sulkunen et al., 2019). Support and treatment for gamblers and their significant others should be easily available, but it is the gambling products and the gambling market that should be regarded as the main problems, rather than the individual gamblers. It should thus be the responsibility of the state and the gambling market to protect the consumer from gambling harms (see also Livingstone \& Rintoul, 2020). To seriously address the social, economic, and health-related harms caused by pachinko and pachislot in Japan, these products need to be acknowledged and problematized as "gambling" and not merely described as "entertainment" or "gaming" in the Japanese legislation (Takiguchi \& Kawanishi, 2020; Brooks et al., 2008).

\section{Limitations}

The participants' stories are shaped by and given meaning through the interaction within the social and cultural context, in the specific interview situation and in Japanese society in general. The focus in this analysis was thus to study how the participants relate to their experiences and manage impressions of self and others, shaped by what is possible to express within the 
treatment and self-help settings in which they were recruited. The analysis would have benefited from more interviews, also including gamblers who had not sought help for their predicaments, and significant others, which could have clarified their role in the recovery process. The translation process from Japanese to English language may have changed some meanings of shame, guilt, and stigma the participants attributed to gambling and recovery. Despite these limitations, our analysis contributes to increased understanding of the complexities of gambling problems in Japan. In future research, it would be important to study how self-help groups such as GA and Gam-Anon have been adjusted to fit in the specific Japanese context, and how shame, guilt, and stigma are addressed specifically in these settings. Lastly, we would like to emphasize the value and need of a deeper sociocultural approach towards the pachinko parlor, its environment, visitors and employees, similar to Kate Bedford's research on bingo capitalism (2019), to further understand the regulation of everyday life on the verge of pleasurable consumption and life-destroying addiction.

\section{References}

Adams, P.J. \& Rossen, F. (2012). A tale of missed opportunities: pursuit of a public health approach to gambling in New Zealand. Addiction, 107, 1051-1056. https://doi.org/10.1111/j.1360-0443.2012.03800.x

Akiyama, K., Shinohara, K., Sakamoto, A., Shoun, A., Komoto, Y., Sato, T., Nishimura, N., Ishida, H., \& Makino, N. (2019). Risk of gambling disorder based on participation level for the Japanese gambling games of pachinko and pachislot: A preliminary study. International Gambling Studies, 19(1), 125-147. https://doi.org/10.1080/14459795.2018.1520908

Alexius, S. (2017). Assigning responsibility for gambling-related harm: scrutinizing processes of direct and indirect consumer responsibilization of gamblers in Sweden. Addiction Research \& Theory, 25(6), 462-475. https://doi.org/10.1080/16066359.2017.1321739

Ando, S., Yamaguchi, S., Aoki, Y., \& Thornicroft, G. (2013). Review of mental-health-related stigma in Japan. Psychiatry and Clinical Neurosciences, 67(7), 471-82. https://doi.org/10.1111/pcn.12086

Bedford, K. (2019). Bingo capitalism - the law and political economy of everyday gambling. Oxford Scholarship Online. https://doi.org/10.1093/oso/9780198845225.001.0001

Blaszczynski, A., Collins, P., Fong, D. et al. (2011). Responsible gambling: General principles and minimal requirements. Journal of Gambling Studies, 27, 565-573. https://doi.org/10.1007/s10899-010-9214-0

Blumer, H. (1969). Symbolic interactionism: Perspective and method. Prentice Hall.

Brooks, G., Ellis, T., \& Lewis, C. (2008). Pachinko: A Japanese addiction? International Gambling Studies, 8(2), 193-205. https://doi.org/10.1080/14459790802168958

Campbell, C., \& Smith, G. (2003). Gambling in Canada, from vice to disease to responsibility: A negotiated history. Canadian Bulletin of Medical History, 20(1), 121-149. https://doi.org/10.3138/cbmh.20.1.121

Carroll, A., Rodgers, B., Davidson, T., \& Sims, S. (2013). Stigma and help-seeking for gambling problems. Australian National University. http://dx.doi.org/10.11575/PRISM/9777

Cassidy, R. (2020). Vicious games: capitalism and gambling. Pluto Press.
Cooley, C. H. (1992). Human nature and the social order. Scribner's.

Corrigan, P. (2004). How stigma interferes with health care. American Psychologist, 59(7), 614-625. https://doi.org/10.1037/0003066X.59.7.614

Doi, T. (1986). The anatomy of self. Kodansha International.

Esping-Andersen G. (1997). Hybrid or unique? The Japanese welfare state between Europe and America. Journal of European Social Policy, 7(3), 179-189. https://doi.org/10.1177/095892879700700301

Francis, L. \& Livingstone, C. (2021). Discourses of responsible gambling and gambling harm: observations from Victoria, Australia. Addiction Research \& Theory, 29(3), 212-222. https://doi.org/10.1080/16066359.2020.1867111

GA Japan Information Center. (n.d.). Gamblers Anonymous Japan region. Retrieved October 12, 2020 from http://www.gajapan.jp/

Gam-Anon. (n.d.). Gam-Anon groups Japan. Retrieved October 12, 2020 from http://www.gam-anon.jp/group

Gaming Technologies Association. (2020). World count of gaming machines 2019. https://gamingta.com/the-world-count-ofgaming-machines-2019/

Goffman, E. (1963). Stigma: Notes on the management of spoiled identity. Penguin.

Government of Japan. (2019). Basic plan for promoting gambling addiction measures 2019 (in Japanese). https://www.kantei.go.jp/jp/singi/gambletou_izonsho/pdf/kiho n_keikaku_honbun.pdf

Government of Japan. (2020). The implementation process during the first year of Reiwa (in Japanese).

https://www.kantei.go.jp/jp/singi/gambletou_izonsho/pdf/r01 shintyoku jyoukyou.pdf

Higuchi, S. (2020). Casino opening, gambling disorder, and newly developed legislative measures targeting the disorder in Japan. Psychiatry and Clinical Neuroscience, 74, $165-165$. https://doi.org/10.1111/pcn.12977

Higuchi, S., \& Matsushita, S. (2017, September 29). Epidemiological survey on gambling addictions in Japan: An interim report on the investigation (in Japanese). National Hospital Organization Kurihama Medical and Addiction Center. https://kurihama.hosp.go.jp/about/pdf/info 20171004.pdf

Hing, N., Nuske, E., Gainsbury, S., \& Russell, A. (2016a). Perceived stigma and self-stigma of problem gambling: Perspectives of people with gambling problems. International Gambling Studies, 16(1), 31-48. https://doi.org/10.1080/14459795.2015.1092566

Hing, N., Nuske, E., Gainsbury, S., Russell, A., \& Breen, H. (2016b). How does the stigma of problem gambling influence help-seeking, treatment and recovery? A view from the counselling sector. International Gambling Studies, 16(2), 263-280. https://doi.org/10.1080/14459795.2016.1171888

Hing, N., Russell, A., Nuske, E., \& Gainsbury, S. (2015). The stigma of problem gambling: Causes, characteristics and consequences. Victorian Responsible Gambling Foundation. https://responsiblegambling.vic.gov.au/documents/351/HingStigma-of-problem-gambling-2015.pdf

Horiuchi, Y., Sakamoto, A., Akiyama, K., Shoun, A., Nishimura, N., Shinohara, K., Komoto, Y., Sato, T., Ishida, H. \& Makino, N. (2018). Prevalence of pachinko-pachislot playing disorder and the characteristics of individuals with the disorder: Analysis of national pachinko/pachislot survey results. Open Journal of Psychiatry, 8(2), 120-130. https://doi.org/10.4236/ojpsych.2018.82011

Imai, A. (2018). Casinos in Japan. Lancet Psychiatry, 5(10), e25. https://doi.org/10.1016/S2215-0366(18)30306-7

Ino, H., Iyama, A., \& Takahashi, A. (2020). Online survey of gambling participation and problem gambling in Chiba city: Problem gambling rates may be strongly influenced by the administration mode. Journal of Gambling Studies, 36(1), 957978. https://doi.org/10.1007/s10899-020-09958-x 
Ito, K. \& Crutcher, P. A. (2014). Popular mass entertainment in Japan: manga, pachinko, and cosplay. Symposium: Signs, Symbols, and Semiotics, 51, 44-48.

https://doi.org/10.1007/s12115-013-9737-y

Japan Productivity Center. (2020). White paper on leisure industry 2019. Retrieved November 13, 2020 from https://www.segasammy.co.jp/english/media/file/ir/managem ent/market/market data.pdf

Japan Productivity Center. (2021). White paper on leisure industry 2020. Retrieved June 4, 2021 from https://www.segasammy.co.jp/

Kamimura, S. (2020, February 20). Gambling addiction now covered by Japanese public health insurance. Inside Asian Gaming. https://www.asgam.com/index.php/2020/02/20/gamblingaddiction-now-covered-by-japanese-public-health-insurance/

Kato, H. \& Goto, R. (2018). Geographical accessibility to gambling venues and pathological gambling: an econometric analysis of pachinko parlours in Japan. International Gambling Studies, 18(1), 111-123. https://doi.org/10.1080/14459795.2017.1383503

Kohler Riessman, C. (2006). Narrative analysis. In V. Jupp (Ed.), The Sage dictionary of social research methods (pp. 186-189). Sage.

Komoto, Y. (2014). Factors associated with suicide and bankruptcy in Japanese pathological gamblers. International Journal of Mental Health and Addiction, 12(5), 600-606. https://doi.org/10.1007/s11469-014-9492-3

Komoto, Y. (2015). Brief intervention based on Naikan therapy for a severe pathological gambler with a family history of addiction: Emphasis on guilt and forgiveness. Asian Journal of Gambling Issues and Public Health, 5(2), 1-8. https://doi.org/10.1186/s40405-015-0007-3

Lebra, T. (1983). Shame and guilt: A psychocultural view of the Japanese self. Ethos, 11(3), 192-209.

Livingstone, C., \& Rintoul, A. (2020). Moving on from responsible gambling: A new discourse is needed to prevent and minimise harm from gambling. Public Health, 184, 107-112. https://doi.org/10.1016/j.puhe.2020.03.018

Loy, J., Grüne, B., Braun, B., Samuelsson, E., \& Kraus, L. (2019). Helpseeking behaviour of problem gamblers: A narrative review. Sucht, 64, 259-272. https://doi.org/10.1024/0939-5911/a000560

Lundberg, O., Yngwe, A. M., Stjärne, M. K., Elstad, J. I., Ferrarini, T., Kangas, O., Norström, T., Palme, J., Fritzell, J., and NEWS Nordic Expert Group. (2008). The role of welfare state principles and generosity in social policy programmes for public health: An international comparative study. The Lancet, 372, 1633-1640. https://doi.org/10.1016/S0140-6736(08)61686-4

Manzenreiter, W. (2013). Playing against all odds: Pachinko and the culture of risk-taking in Japan's crisis economy. Leisure Studies, 32(3), 283-298. https://doi.org/10.1080/02614367.2011.652156

Matsushita, S., Nitta, C., \& Toyama, T. (2021). Fact-finding survey on gambling disorders and gambling-related problems. Reiwa 2nd year research project on addiction. (In Japanese). https://www.ncasa-japan.jp/docs/

Miceli, M., \& Castelfranchi, C. (2018). Reconsidering the differences between shame and guilt. Europe's Journal of Psychology, 14(3), 710-733. https://doi.org/10.5964/ejop.v14i3.1564

Miller, H. E., \& Thomas, S. (2017). The "walk of shame": a qualitative study of the influences of negative stereotyping of problem gambling on gambling attitudes and behaviours. International Journal of Mental Health and Addiction, 15(6), 1284-1300. https://doi.org/10.1007/s11469-017-9749-8

Miller, H. E., \& Thomas, S. L. (2018). The problem with 'responsible gambling': impact of government and industry discourses on feelings of felt and enacted stigma in people who experience problems with gambling. Addiction Research \& Theory, 26(2), 85 94. https://doi.org/10.1080/16066359.2017.1332182

Ministry of Health, Labour and Welfare. (2019). The outline of social welfare (public assistance) system. (In Japanese) https://www.mhlw.go.jp/content/12002000/000488808.pdf
Mori, T., \& Goto, R. (2020). Prevalence of problem gambling among Japanese adults. International Gambling Studies, 20(2), 231-239. https://doi.org/10.1080/14459795.2020.1713852

Moriyama, N. (2016). Ethical, legal, and social issues (ELSI) in gambling disorder and its treatment. Brain Nerve, 68(10), 11671176. https://doi.org/10.11477/mf.1416200570

Oka, T. (2013). Self-help groups in Japan: Historical development and current issues. International Journal of Self-Help and Self-Care, 7(2), 217-232. https://doi.org/10.1080/10852359409511197

Orford, J. (2019). The gambling establishment: challenging the power of the modern gambling industry and its allies. Routledge.

Penal Code. (n.d.) (Keiho) Penal code act no. 45 of 1907. Retrieved November 16, 2020 from http://www.japaneselawtranslation.go.jp/law/detail_main?re= \&vm $=02 \& i d=1960$

Raylu, N., \& Oei, T. (2004). Role of culture in gambling and problem gambling. Clinical Psychology Review, 23, 1087-1114. https://doi.org/10.1016/j.cpr.2003.09.005

Reith, G. (2007). Gambling and the contradictions of consumption: A genealogy of the 'pathological' subject. American Behavioral Scientist, 51(1), 33-55. https://doi.org/10.1177/0002764207304856

Roberts, J., \& Johnson, T. (2016). Problem gambling: How Japan could actually become the next Las Vegas. UNLV Gaming Law Journal, 6(2), 176-198.

Rockwell, G. \& Amano, K. (2019). Pachinko: a case study in hybrid physical and virtual interface. Journal of the Japanese Association for Digital Humanities, 4(1), 72-89. https://doi.org/10.17928/jjadh.4.1_72

Room. R. (2005). Stigma, social inequality and alcohol and drug use. Drug and Alcohol Review, 24, 143-155. https://doi.org/10.1080/09595230500102434

Rose, N. (1999). Governing the soul: The shaping of the private self. Free Association Books.

Samuelsson, E., Sundqvist, K., Binde, P. (2018). Configurations of gambling change and harm: Qualitative findings from the Swedish longitudinal gambling study (Swelogs). Addiction Research \& Theory, 26, 514-524. https://doi.org/10.1080/16066359.2018.1448390

Saraceno, C. (2016). Varieties of familialism: Comparing four southern European and East Asian welfare regimes. Journal of European Social Policy, 26(4), 314-326. https://doi.org/10.1177/0958928716657275

Scheff, T. (1990). Microsociology: Discourse, emotion and social structure. The University of Chicago Press.

Scheff, T. (2003). Shame in self and society. Symbolic Interaction, 26(2), 239-262. https://doi.org/10.1525/si.2003.26.2.239

Scheff, T. (2013). Goffman on emotions: The pride-shame system. Symbolic Interaction, 37(1), 108-121. https://doi.org/10.1002/symb.86

Schüll, N. (2012). Addiction by design: Machine gambling in Las Vegas. Princeton University Press. https://doi.org/10.1515/9781400834655

So, R., Matsushita, S., Kishimoto, S., \& Furukawa, T. A. (2019). Development and validation of the Japanese version of the problem gambling severity index. Addictive Behaviors, 98, 1-6 (Article 105987). https://doi.org/10.1016/j.addbeh.2019.05.011

Song, T. R. (2009). Shame and guilt in the Japanese culture: $A$ study of lived experiences of moral failures of Japanese emerging generation and its relation to the church missions in Japan. [PhD. Thesis, Trinity International University]. ProQuest Dissertations Publishing.

Sulkunen, P., Babor, T. F., Örnberg, J. C., Egerer, M., Hellman, M., Livingstone, C., Marionneau, V., Nikkinen, J., Orford, J., Room, R., \& Rossow, I. (Eds.). (2019). Setting limits: Gambling science, and public policy. Oxford University Press. https://doi.org/10.1093/oso/9780198817321.001.0001 
Suurvali, H., Cordingley, J., Hodgins, D., \& Cunningham, J. (2009). Barriers to seeking help for gambling problems: A review of the empirical literature. Journal of Gambling Studies, 25(3), 407-424. https://doi.org/10.1007/s10899-009-9129-9

Suzuki, M., Ito, M., Ishida, M., Nihei, N., \& Maruyama, M. (2010). Individualizing Japan: Searching for its origin in first modernity. BJS, The British Journal of Sociology, 61(3), 513-538. https://doi.org/10.1111/j.1468-4446.2010.01324.x

Swanton, T. B., Gainsbury, S., \& Blaszczynski, A. (2019). The role of financial institutions in gambling. International Gambling Studies, 19(3), 377-398. https://doi.org/10.1080/14459795.2019.1575450

Takiguchi, N., \& Kawanishi, Y. (2020, February 12). Japan gambles on casinos to fix its economic woes. East Asia Forum. https://www.eastasiaforum.org/2020/02/12/japan-gambles-oncasinos-to-fix-its-economic-woes/

Takiguchi, N., \& Rosenthal, R. J. (2011). Problem gambling in Japan: A social perspective. Electronic Journal of Contemporary Japanese Studies, $11(1)$. N.p. https://japanesestudies.org.uk/articles/2011/Takiguchi.html

Tanabe, H. (2010). Pathological gambling and suicide. Japanese Journal of Psychiatric Treatment, 25(2), 223-229.

Tanaka, N. (2018, January 4). Gambling addiction in the land of pachinko. Nippon.com. https://www.nippon.com/en/currents/d00367/

Tangney, J. (2002). Shame and guilt. In D. Levinson (Ed.), Encyclopedia of crime and punishment (pp. 1492-1494). Sage. https://www.doi.org/10.4135/9781412950664.n388

Thomas, R., Deighton, R., Mizuno, M., Yamaguchi, S., \& Fujii, C. (2020). Shame and self-conscious emotions in Japan and Australia: Evidence for a third shame logic. Culture \& Psychology, 26(3), 622-638. https://doi.org/10.1177/1354067X19851024

Tomida, K., \& Hisanaga, R. (2020, January 12). Panel urges that public insurance cover therapy for gambling addicts. The Asahi Shimbun. http://www.asahi.com/ajw/articles/13056287

Törrönen, J., Samuelsson, E., Gunnarsson, M. (2020). Online gambling venues as relational actors in addiction: Applying the actornetwork approach to life stories of online gamblers. International Journal of Drug Policy, 85, 1-9 (Article 102928). https://doi.org/10.1016/j.drugpo.2020.102928

Toyama, T., Nakayama, H., Takimura, T., Yoshimura, A., Maesato, H., Matsushita, S., Osaki, Y., \& Higuchi, S. (2014). Symposium SY174. Prevalence of pathological gambling in Japan: Results of national surveys of the general adult population in 2008 and 2013. Alcohol and Alcoholism, 49(1), i17. https://doi.org/10.1093/alcalc/agu052.75

Yakovenko, I., \& Hodgins, D. (2018). A scoping review of co-morbidity in individuals with disordered gambling. International Gambling Studies, 18(1), 43-172. https://doi.org/10.1080/14459795.2017.1364400

Yi, S., \& Kanetkar, V. (2011). Coping with guilt and shame after gambling loss. Journal of Gambling Studies, 27(3), 371-387. https://doi.org/10.1007/s10899-010-9216-y

Yokomitsu, K., Kamimura, E., \& Somatori, K. (2020). Validation of the Japanese version of the pathological gambling adaptation of the Yale-Brown Obsessive-Compulsive Scale (PG-YBOCS-J) among subclinical Japanese gamblers. International Journal of Mental Health and Addiction 19, 1716-1728. https://doi.org/10.1007/s11469-020-00258-4

Yokomitsu, K., Sakai, T., Irie, T., Tayama, J., Furukawa, H., Himachi, M., Kanazawa, J., Koda, M., Kunisato, Y., Matsuoka, H., Takada, T., Takahashi, F., Takahashi, T., \& Osawa, K. (2019). Gambling symptoms, behaviors, and cognitive distortions in Japanese university students. Substance Abuse Treatment, Prevention, and Policy, 14(51), 1-7. https://doi.org/10.1186/s13011-019-0230-5

\section{Funding and Conflict of Interest Statement}

Funding for the study was provided by The Scholarship Foundation for Studies of Japanese Society by the program grant "Responding to and Reducing Gambling Problems Studies" (REGAPS; FORTE no 2016-07091), by the postdoc project "Dilemmas of Help-seeking - Needs, Experiences, and Barriers in Contact with Care in the Case of Gambling and Alcohol Problems" (FORTE no 2016-00286), and by the project "Addiction" as a changing pattern of relationships: Comparing autobiographical narratives about different dependencies' (VR no 2018-01297). The authors declare they have no competing interests.

\section{Acknowledgements}

The authors are grateful to the interviewees who generously shared their stories, as well as to the anonymous reviewers and members of the REGAPS research group who commented on earlier drafts of this article.

\section{Author Details}

Eva Samuelsson ( $\mathrm{PhD}$ in social work) is a Senior Lecturer at the Department of Social Work, and researcher at the Department of Public Health Sciences/SoRAD, Stockholm University. She conducts research on help-seeking processes and organization of support and treatment for people with substance use and gambling problems.

Jukka Törrönen (PhD in sociology) is Professor at the Department of Public Health Sciences/SoRAD, Stockholm University. He has a long-term interest in substance use research, theoretical sociology, comparative studies, new materialism, and qualitative methods. His work has focused on disorderly public drinking, young people's drinking cultures, women's substance use, and diverse forms of dependence problems.

Chiyoung Hwang is a $\mathrm{PhD}$ candidate at the Department of Health Promotion and Human Behavior, Graduate School of Medicine, Kyoto University. She is also a research fellow (DC2) of Japan Society for the Promotion Science. Her dissertation deals with gambling-related problems and homelessness in Japan.

Naoko Takiguchi, PhD, is a Professor of Sociology at Otani University and board chair of Kyoto Mac Addiction Center, Japan. She serves on the committee of Promoting Measures against Gambling Addiction in Kyoto and Osaka. She has provided a gambling-specific education program at correction facilities and invited speakers at numerous addiction seminars open to the public. Her current research topic is the social cost of gambling harms in Japan.

\section{ORCID}

Eva Samuelsson

https://orcid.org/0000-0002-0856-9854 Jukka Törrönen (iD

http://orcid.org/0000-0003-2473-6330 